\title{
Tracheal laceration during intubation of a double-lumen tube and intraoperative fiberoptic bronchoscopic evaluation through an LMA in the lateral position -A case report-
}

\author{
Joohee Kim, Taewan Lim, and Jae-Hyon Bahk \\ Department of Anesthesiology and Pain Medicine, Seoul National University Hospital, Seoul, Korea
}

A 76-year-old, 148-cm woman was scheduled for right upper lobectomy. A $32 \mathrm{Fr}$ left-sided double lumen tube was placed using a conventional technique. Despite several attempts under fiberoptic bronchoscope-guidance, we could not locate the double lumen tube properly. We thus decided to proceed with the bronchial tube in the right mainstem bronchus. During surgery, 8-cm-long laceration was noted on the posterolateral side of the trachea. To check the possibility of laceration of the proximal trachea, the double lumen tube was changed to an LMA for use as a conduit for fiberoptic bronchoscopic evaluation in the lateral position. A plain endotracheal tube with the cuff modified and collapsed was re-intubated after evaluation. And then she was transferred to SICU. (Korean J Anesthesiol 2011; 60: 285-289)

Key Words: Fiberoptic bronchoscope, Intubation, Laceration, LMA, Trachea.

An endobronchial double-lumen tube is often used as a technique to maintain single-lung ventilation during thoracic surgery. However, it can cause a tracheal rupture due to the relatively large external diameter and stylet that are often used $[1,2]$. A tracheal rupture after intubation is a rare complication, but is critical when diagnosis and treatment are late and it is important to manage the airway before and after the treatment. In the event of uncertainty regarding a tracheal rupture, we recommend using a fiberoptic bronchoscope to assess the degree and range of tracheal rupture and develop a treatment plan. In addition, to prevent complications due to the increased airway pressure caused by the endotracheal tube or mechanical ventilation, it is ideal to conduct extubation early to allow the patient to maintain spontaneous respiration. However, in most cases, mechanical ventilation is required after an operation for a tracheal rupture [3]. From a case study of a 76-year-old female patient who suffered a tracheal rupture caused by intubation by a endobronchial double-lumen tube while having a right upper lobectomy and esophageal enucleation, we, the authors, discuss here the overall management of a tracheal rupture that

Received: July 27, 2010. Revised: October 6, 2010. Accepted: October 8, 2010.

Corresponding author: Jae-Hyon Bahk, M.D., Department of Anesthesiology and Pain Medicine, Seoul National University Hospital, 101, Daehak-ro, Yungun-dong, Jongro-gu, Seoul 110-744, Korea. Tel: 82-2-2072-2467, Fax: 82-2-747-5639, E-mail: bahkjh@snu.ac.kr

(c) This is an open-access article distributed under the terms of the Creative Commons Attribution Non-Commercial License (http:// creativecommons.org/licenses/by-nc/3.0/), which permits unrestricted non-commercial use, distribution, and reproduction in any medium, provided the original work is properly cited. 
was found during the operation, the application of a fiberoptic bronchoscope using the laryngeal mask airway as the pathway to verify restoration of the tracheal rupture, and the use of an endotracheal tube that is specially designed to maintain and manage the airway after an operation.

\section{Case Report}

A 76-year-old female patient, $148 \mathrm{~cm}$ in height and $55 \mathrm{~kg}$ in weight, was scheduled for a right upper lobectomy and esophageal enucleation. The patient was taking anti-hypertensive medication for hypertension, and we could not find any specific abnormalities through examinations before the surgery, including a blood test, electrocardiography, electrocardiography, chest CT (computed tomography) and broncoscopic examination.

The patient was brought to the operating room without premedication. Her initial vital signs were as follows: blood pressure of 179/72 mmHg; heart rate of $94 \mathrm{bpm}$; and saturation of $98 \%$. Denitrogenation was done with $100 \%$ oxygen, and general anesthesia was induced with lidocaine $30 \mathrm{mg}$, fentanyl $100 \mu \mathrm{g}$, propofol $100 \mathrm{mg}$, rocuronium $50 \mathrm{mg}$ and sevoflurane 8 vol\%. After obtaining an appropriate depth of anesthesia, she was intubated with a 32 Fr left-sided double-lumen tube (Broncho-cath ${ }^{\circledR}$, Mallinckrodt Medical Ltd., Ireland) without difficulty using a conventional technique and then checked the location of the tube via auscultation. But as the left bronchial tip of the double-lumen tube was inserted to the right main bronchus, we tried to move it to the right position using a fiberoptic bronchoscope with an external diameter of $3.1 \mathrm{~mm}$

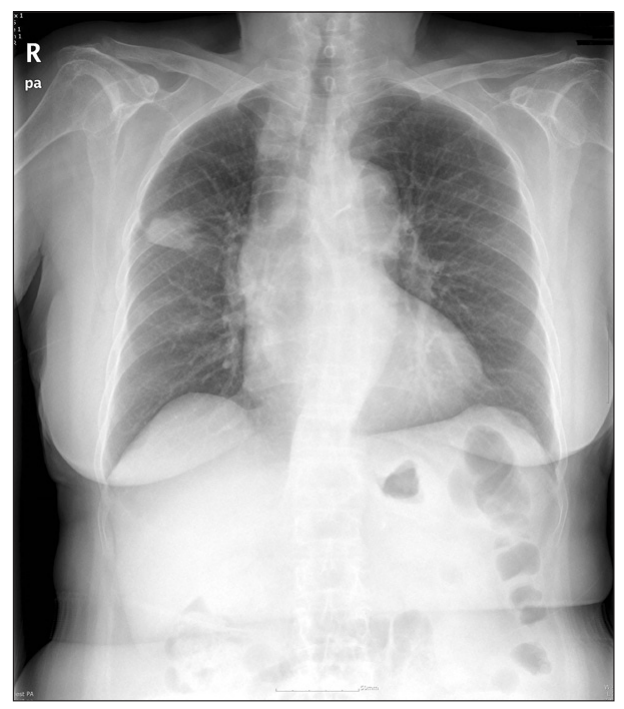

Fig. 1. The preoperative chest X-ray shows the tracheal deviation to the right side.
(LF-DP, Olympus, Japan). Placing the fiberoptic bronchoscope in the left main bronchus was not difficult, but when we pushed the tube, the left bronchial tip slid into the right main bronchus. We tried using another method; the stylet was not removed just after the tip of the tube passed the vocal cord and was retained for the entire intubation procedure [4]. However, it could not enter the left main bronchus. In the image of the patient's chest radiography, the trachea was deviated on the right side (Fig. 1) and in the computed tomography, we noted that the internal diameter of the trachea, at the point of the right main bronchus and the left main bronchus was $11.25 \mathrm{~mm}, 12.5 \mathrm{~mm}$ and 8.6 $\mathrm{mm}$, respectively (Fig. 2). Inevitably, we decided to proceed with the operation while leaving the left bronchial tip of the double-lumen tube inserted in the right main bronchus. At the time, the saturation of the patient was maintained at $99-100 \%$ and her vital signs were stable.

During hemostasis after the right upper lobectomy, the surgeon observed that, there was a bulging lesion of a mediastinal pleura near the right second rib. When the surgeon incised it, an air leak was observed at the carina. At the time, we also found that the tidal volume was decreased. We therefore increased the inspiratory pressure of the pressure-controlled ventilation to $24 \mathrm{~cm} \mathrm{H} \mathrm{H}_{2} \mathrm{O}$ to hold the tidal volume at $190-220 \mathrm{ml}$. The surgeon noted that the tracheal balloon of double-lumen tube was visible through the damaged trachea. As it would cause an obstruction when restoring the trachea, we fixed it after withdrawing it by about $2 \mathrm{~cm}$ of the endobronchial doublelumen tube and started two-lung ventilation. The tidal volume, which was maintained at more than $300 \mathrm{ml}$ during single-lung ventilation, decreased to $40 \mathrm{ml}$, whereas saturation fell to $90 \%$. The anesthesia machine showed the warning sign of "circuit leak" on its monitor. However, we tried manual ventilation with $100 \% \mathrm{O}_{2}$ and maintained permissive hypercapnia so as

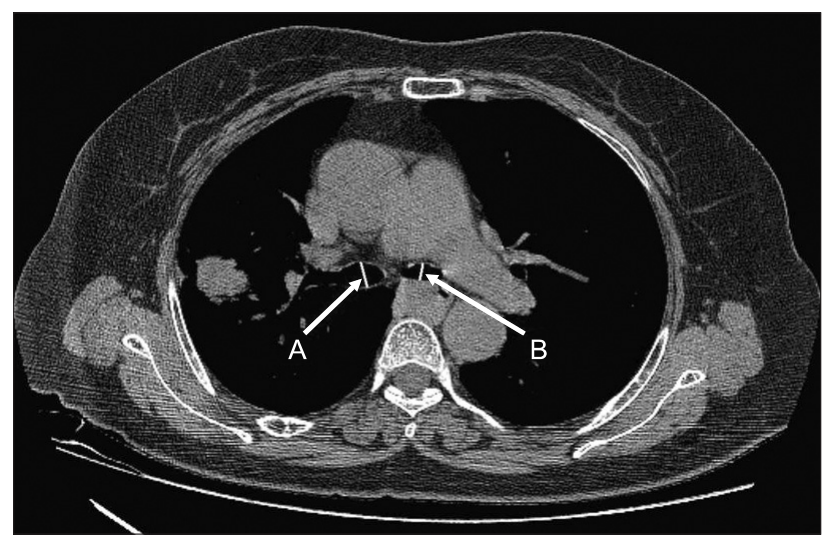

Fig. 2. The preoperative chest computed tomography scan. (A) the diameter of the right main bronchus, $12.5 \mathrm{~mm}$. (B) the diameter of left main bronchus, $8.6 \mathrm{~mm}$. 


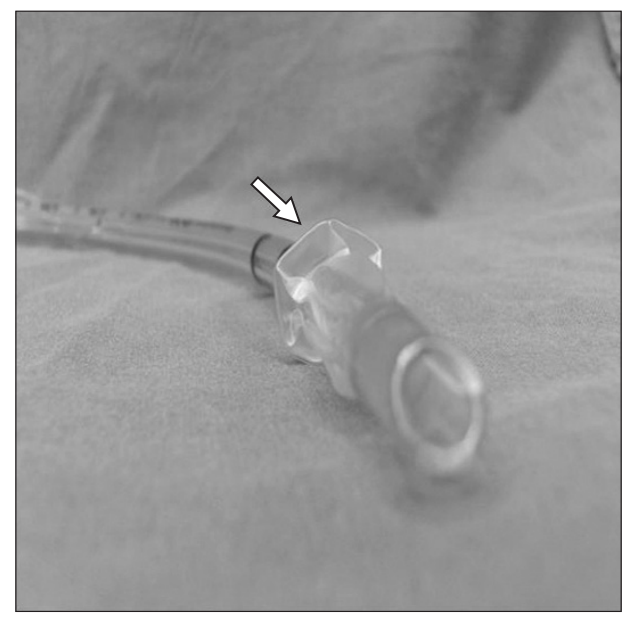

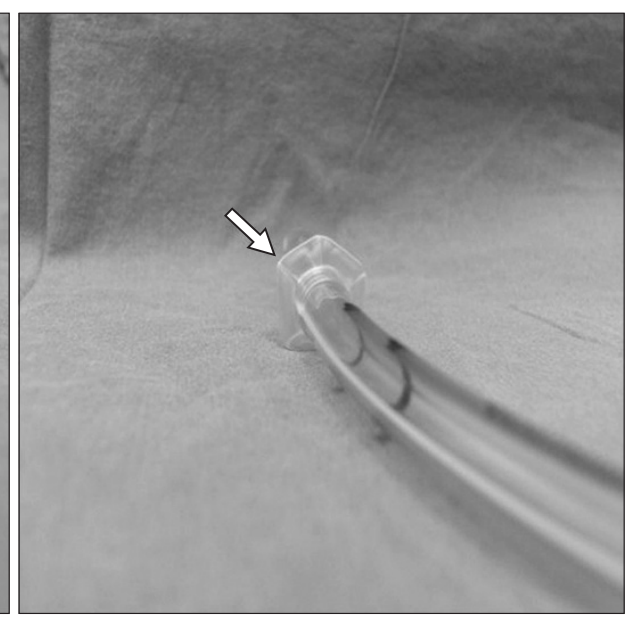

Fig. 3. The cuff of an internal diameter $7.0 \mathrm{~mm}$ endotracheal tube was modified during deflation to make a frill-like fold (arrows) at the proximal end. not to disturb the repair of the trachea. We restored a tracheal laceration of $8 \mathrm{~cm}$ from the initial segment of the carina up to 5 cm under the vocal cord.

Repair of the intrathoracic trachea was completed, but checking the tracheal laceration in the proximal part remained. We decided to conduct a fiberoptic bronchoscopic evaluation. In the left lateral decubitus position, we extubated an endobronchial double-lumen tube and inserted a laryngeal mask airway (LMA-CLASSIC ${ }^{\mathrm{TM}}$, Intavent Orthofix, UK) to use it as a pathway for the fiberoptic bronchoscopic evaluation. The evaluation revealed that the repair was complete, as the proximal part of the tracheal laceration was located up to $5 \mathrm{~cm}$ below the vocal cord. After the surgery, intubation was done using an ID $7.0 \mathrm{~mm}$ single-lumen tube with a deflated cuff (Fig. 3) with the patient in a supine position and then fixed the tube so that the cuff was located in the area right under the vocal cord, where it was not damaged. By carrying out a leakage test, we checked that there was no air leak around the cuff at an inspiratory pressure of $20 \mathrm{~cm} \mathrm{H}_{2} \mathrm{O}$. The patient was then transferred to the intensive care unit (ICU). In the ICU, tidal volume of the patient was kept at around $300 \mathrm{ml}$ with an inspiratory pressure of $10 \mathrm{~cm} \mathrm{H}_{2} \mathrm{O}$ by synchronized intermittent mandatory ventilation (SIMV). Extubation was performed in the ICU about 14 hours after the surgery.

On the second postoperative day, the patient was transferred to the general ward. On the fourth postoperative day, a fiberoptic bronchoscopic evaluation was performed. In the result, we could not find any abnormities in the left main bronchus, while the internal diameter of the right main bronchus appeared a bit narrower due to edema, hyperemia and granulation just below the carina. However, there was no problem with the repaired lesion. On the eleventh postoperative day, we carried out another fiberoptic bronchoscopic evaluation and checked that the patient was recovered completely. She was discharged on the twelfth postoperative day without complications.

\section{Discussion}

A double-lumen tube has been widely used for lung separation procedures due to the ease of placement and because it serves as a rapid means of switching between single-lung ventilation and two-lung ventilation. It can also be used with single-lung ventilation simply by auscultation without a fiberoptic bronchoscope and if it is positioned appropriately, its position will not be changed easily during the surgical procedures. Nonetheless, when there is some variation in the anatomical structure of the airway, intubation and positioning is difficult, and because the volume and external diameter of this type of tube is relatively large, it is not appropriate to continue mechanical ventilation for a long time $[5,6]$. In addition, a tracheal injury can occur due to intubation with an endobronchial double-lumen tube, together with tracheal irritation, pharyngitis and an occasionally tracheobronchial rupture as well. The frequency of a tracheal rupture due to intubation with an endobronchial double-lumen tube is known to be less than $1 \%$ [2]. It mainly occurs as a longitudinal laceration at the membranous portion of the trachea [7].

Major factors for tracheal rupture include an inexperienced professional during the medical procedure, repetitive attempts, inappropriate use of the stylet, an over-expanded cuff, inappropriate selection of the tube size, abnormal location of the tube, sudden movement of the patient, excessive coughing, weakened membrane structure of the trachea due to steroid or radiation therapy, chronic obstructive pulmonary disease (COPD), and tracheomalacia. Moreover, other contributing factors include a short stature, obesity, use of an endobronchial double-lumen tube, patient aged older than 50 years old, percutaneous tracheostomy and more. In addition, it more often occurs in women $[8,9]$. As in this case study, with a relatively short trachea of a patient who has a short stature, it can occur when the tube is positioned in the distal position and 
when the size is not appropriate. In the computed tomography of this case study, we found that the diameter of the left main bronchus of the patient was $8.6 \mathrm{~mm}$. As the external diameter of the end of the $32 \mathrm{Fr}$ double-lumen tube is $8.2 \mathrm{~mm}$, selection of the 32 Fr double-lumen tube can be considered as an appropriate choice. However, despite the fact that we used a fiberoptic bronchoscope, as we induced anesthesia for about 1 hour and 20 minutes and repetitively attempted intubation, we thought that it should have induced a tracheal rupture. In addition, although the stylet should be removed after it passes through the vocal cords, we removed it at the lower level of the trachea during the intubation process as the end of the tube should enter into the left side; this can be considered as another cause of a tracheal rupture. Thus, although it was a right upper lobectomy, it would have been better to conduct single-lung ventilation using a single-lumen tube and a bronchial blocker, rather than repetitively attempting intubation with the doublelumen tube.

Tracheal ruptures are rare but can be life-threatening. Immediate recognition and adequate treatment are very important in managing this situation. A high level of suspicion based on clinical manifestations is important in diagnoses [2]. If the rupture is caused by intubation, if we start mechanical ventilation, clinical symptoms of subcutaneous emphysema, pneumomediastinum and bleeding will start rapidly and pneumothorax can develop when the pleural cavity is affected by a tracheal rupture. However, as in this case, as clinical symptoms may not be recognized soon after the rupture and may only be found after a long time, it is necessary to pay attention [10]: when there is blood in the tracheal tube, we have to check the trachea immediately [2]. Chest computed tomography will be helpful in finding hemomediastinum or pneumomediastinum whereas esophageal endoscopy should be conducted when communication between the trachea and esophagus is in doubt. Because a fiberoptic bronchoscope can determine the depth and extent the lesion, it is necessary in developing a treatment plan and is also useful for checking for complications after the treatment, such as granulation or tracheal stenosis [10]. In this case study, before checking for subcutaneous emphysema or pneumothorax, the surgeon observed bulging of the mediastinal pleura near the right second rib and when he incised it, an air leak was observed from the carina region, which indicated a tracheal rupture. In addition, a decrease in the tidal volume and saturation during mechanical ventilation increased the possibility of a tracheal rupture.

Management of the airway during the repair of a tracheal rupture require providing safe ventilation and adequate surgical access. Above all, no additional tracheal damage should ensue during inspiration. There are some methods of ventilation including manual jet ventilation, high-frequency positive pressure ventilation, high-frequency jet ventilation, distal tracheal intubation, spontaneous respiration, and cardiopulmonary bypass $[11,12]$. A bridging technique is recommended for mechanical ventilation during repair of a tracheal rupture. The tube is advanced distal to the ruptured site with a minimally inflated cuff, which will not create pressure on the damaged tissue. At this time, a small amount of air leakage is acceptable [13]. However, in this case study, as the location of the rupture was near the carina, we worried about additional damage that could occur during the positioning of the double-lumen tube to the distal part than the ruptured part. Thus, we unfastened the cuff of the double-lumen tube, and we rapidly sutured the distal part of the rupture as withdrew the tube. At that time, respiratory acidosis occurred due to the air leakage, but the vital signs, oxygenation and end-expiratory gas concentration were maintained. As we did not use a Bispectral index (BIS ${ }^{\circledR}$, Aspect Medical Systems Inc., Norwood, MA, USA) machine during the operation, we could not verify the maintenance of anesthesia according to the EEG; however, we did not get any signs of awakening during the operation.

As a tracheal rupture was found during a right upper lobectomy, we could largely check the depth and level of the lesion visually. To check the possibility of tracheal laceration in the larynx and the upper third of the trachea, we changed the endobronchial double-lumen tube to an laryngeal mask airway No. 3, and carried out a fiberoptic bronchoscopic evaluation. The laryngeal mask airway would not increase airway resistance more than the endotracheal tube during the fiberoptic bronchoscopic evaluation, and it maintained appropriate ventilation and inhalation anesthesia. In addition, the LMA allows visualization of the vocal cords and subglottis area while an endotracheal tube must be drawn out in an intubated patient [14].

After an operation for a tracheal rupture, early extubation is recommended under the spontaneous ventilation, as there is the possibility of damage to the mucous layer of the trachea due to the movement of the endotracheal tube and the pressure of the cuff. However, in quite a few cases, mechanical ventilation is necessary after the operation [3], and patients are maintained in the intubated state. Because respiratory acidosis progressed in this patient during the operation, we could not ignore the possibility of re-intubation for mechanical ventilation. We were worried about a re-rupture during an emergency intubation in the ICU, and therefore performed the intubation in the operating room and observed the patient in the ICU. We, the authors, thought of using a single-lumen tube (Fig. 3), the cuff of which was formed to make a frill-like fold at the proximal end, to minimize the pressure over the damaged tracheal mucosa and to provide sealing effect when mechanical ventilation 
assistance was needed. We located the cuff of the tube at the proximal part of the restored area. As there was a sealing effect as the wing-shaped cuff touched the trachea, even with an inspiratory pressure of $20 \mathrm{cmH}_{2} \mathrm{O}$, an air leak did not occur around the cuff. In the fiberoptic bronchoscopic evaluation that was done on the fourth postoperative day, we could not find any specific abnormalities in the location of the cuff.

In conclusion, a tracheal rupture after intubation with an endobronchial double-lumen tube during an operation is rare, but life-threatening, immediate and accurate diagnosis and restoration during the operation are necessary. In addition, immediately after the restoration, an laryngeal mask airway can be used as a conduit for a fiberoptic bronchoscopic evaluation to check the areas of the repaired tracheal laceration while providing adequate mechanical ventilation. Additionally, the authors believe that the specially designed tube will be helpful in maintaining appropriate ventilation while not placing any pressure on the tracheal mucosa after the operation.

\section{References}

1. Massard G, Rouge C, Dabbagh A, Kessler R, Hentz JG, Roeslin N, et al. Tracheobronchial lacerations after intubation and tracheostomy. Ann Thorac Surg 1996; 61: 1483-7.

2. Liu H, Jahr JS, Sullivan E, Waters PF. Tracheobronchial rupture after double-lumen endotracheal intubation. J Cardiothorac Vasc Anesth 2004; 18: 228-33.

3. Mussi A, Ambrogi MC, Menconi G, Ribechini A, Angeletti CA.
Surgical approaches to membranous tracheal wall lacerations. J Thorac Cardiovasc Surg 2000; 120: 115-8.

4. Lieberman D, Littleford J, Horan T, Unruh H. Placement of left double-lumen endobronchial tubes with or without a stylet. Can J Anaesth 1996; 43: 238-42.

5. Cohen E. Methods of lung separation. Curr Opin Anaesthesiol 2002; 15: 69-78.

6. Robinson AR, 3rd, Gravenstein N, Alomar-Melero E, Peng YG. Lung isolation using a laryngeal mask airway and a bronchial blocker in a patient with a recent tracheostomy. J Cardiothorac Vasc Anesth 2008; 22: 883-6.

7. Lampl L. Tracheobronchial injuries. Conservative treatment. Interact Cardiovasc Thorac Surg 2004; 3: 401-5.

8. Chen EH, Logman ZM, Glass PS, Bilfinger TV. A case of tracheal injury after emergent endotracheal intubation: a review of the literature and causalities. Anesth Analg 2001; 93: 1270-1.

9. Kaloud H, Smolle-Juettner FM, Prause G, List WF. Iatrogenic ruptures of the tracheobronchial tree. Chest 1997; 112: 774-8.

10. Hofmann HS, Rettig G, Radke J, Neef H, Silber RE. Iatrogenic ruptures of the tracheobronchial tree. Eur J Cardiothorac Surg 2002; 21: 649-52.

11. Pinsonneault C, Fortier J, Donati F. Tracheal resection and reconstruction. Can J Anaesth 1999; 46: 439-55.

12. Stannard K, Wells J, Cokis C. Tracheal rupture following endotracheal intubation. Anaesth Intensive Care 2003; 31: 588-91.

13. Conti M, Pougeoise M, Wurtz A, Porte H, Fourrier F, Ramon P, et al. Management of postintubation tracheobronchial ruptures. Chest 2006; 130: 412-8.

14. Campos JH. Update on tracheobronchial anatomy and flexible fiberoptic bronchoscopy in thoracic anesthesia. Curr Opin Anaesthesiol 2009; 22: 4-10. 